\title{
Role of noise in human visual perception: A psychophysical study
}

\author{
Ajanta Kundu ${ }^{1}$, Sandip Sarkar ${ }^{2}$ \\ ${ }^{1,2}$ (Applied Nuclear Physics Division, Saha Institute of Nuclear Physics, India)
}

\begin{abstract}
Visual psychophysical studies show that in presence of optimum noise contrast detection sensitivity can be enhanced via a non-linear cooperative effect called stochastic resonance. However, the dependence of the enhanced sensitivity on spatial frequency is not known. In the present study we have addressed this issue by using stochastic resonance as a tool. We have used a two-interval forced-choice (2IFC) protocol, in a visual detection task involving sub-threshold sine grating stimuli at five spatial frequencies $(0.0369,0.1371,0.5456,1.0909$ and 2.0978 cycle/deg). We observed that at each frequency the perceived sensitivity strongly depends on noise strength and attains a peak for an intermediate strength via stochastic resonance. Additionally, the observed peak contrast detection sensitivity at each frequency,thus attained, varies non-monotonically with spatial frequency. We also demonstrate that the qualitative nature of the enhanced detection sensitivity is in good agreement with the human contrast sensitivity function. These results indicate that the human contrast sensitivity behaviour with spatial frequency could be obtained in terms of stochastic resonance.
\end{abstract}

Keywords: contrast detection sensitivity, contrast sensitivity function, human visual system, stochastic resonance, visual psychophysics

\section{Introduction}

Beneficial role of noise in neural computations have been observed at all levels from lower psychological to higher cognitive ones. That is also an extensively investigated phenomenon in physics as well as in biology [1-3] including human sensory systems using psycho-physical methods [4]. Increase in the detect ability of weak signals by the process of stochastic resonance (SR) has been observed, for example, in the crayfish [5], cricket [6] and rats [7]. For complex cognitive systems, SR has been observed in human brain waves [8], ambiguous pattern detection [9], human tactile sensation [10,11] and also in human visual perception [12-15]. Some studies [16-17] point out the possible utility of SR and suggest that it have might offered some survival benefits, which worked in favor of SR in the biological systems.

The direct evidence of detection sensitivity enhancement, via stochastic resonance, is reported for the first time in [12] using psychophysical experiments in the threshold paradigm. They have demonstrated that stochastic resonance, in its simplest threshold paradigm, can be used as tool to measure the detection sensitivity to fine details in noise contaminated images quantitatively and repeatedly. In this experimental paradigm, the original noisy stimulus was depressed beneath an artificial threshold such that the stimulus alone (without noise contamination) could never be visible. The stimulus that is presented to the participants is formed as a result of threshold crossings by the noisy source stimulus. The threshold detector transmitted either ' 0 ' (minimum graylevel) or ' 1 ' (spikes with maximum gray-level) signifying non-occurrence or occurrence of threshold crossing in the positive direction, which resulted in a binary stimulus at its output. These binary stimuli were visually presented for the participants, who were given the task to identify a specific fine structure in the displayed stimuli. For an optimal noise, the participants experienced enhanced detect ability, which degraded for too little or too high noise signifying SR. An evidence of enhanced cortical activity for optimal noise stimulation was also observed in [12] using functional magnetic resonance techniques.

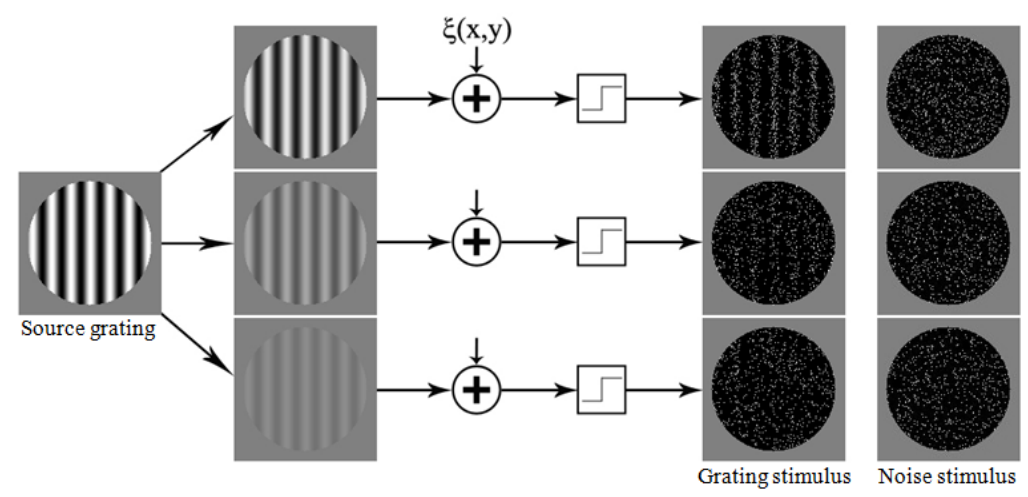


Fig. 1.Stimulus generation procedure is depicted in this figure. Three cases corresponding to three contrast levels are shown in three rows. The image in the first column is the original sine grating. The images in the second column are obtained by decreasing the contrast (top to bottom) of the original grating and in the third column, noise addition procedure is depicted. The threshold operation in the positive direction is represented by the square blocks in the fourth column and the resulting signal stimuli (signal plus noise) are shown in the fifth column. The accompanying noise stimuli with equal number of threshold crossings are shown in the sixth column for which, a noisy source stimulus is input directly to the threshold detector (square block) bypassing stages in column two and three.

An analogous experiment, related to the visual perception of noisy letters, has also been reported later [13], where it is shown that SR played a significant role in the recognition of noisy capitalized letters by the human perceptive system. An evidence of enhanced cortical activity for optimal noise stimulation was observed [18] using functional magnetic resonance techniques. A theoretical investigation about the possible mechanism of stochastic resonance in the light of an extra-classical receptive field is carried out more recently [19]. Enhancement of perceptive threshold contrast sensitivity (inverse of perceptive threshold contrast), via SR, is reported in $[12,13]$, where they have used noisy stimuli that are depressed beneath an artificial threshold. They have also observed that the enhancement varied with noise strength in a non-monotonic fashion.

In all these experiments, the explicit role of spatial frequency of the stimulus or image on our perceptual ability is not addressed. It is not known, how the enhanced sensitivity depend on spatial frequency. To address this issue, in the present work, we have investigated the responese of human observers by using a two-interval forced-choice (2IFC) protocol in a visual detection task involving noisy sine grating stimuli, depressed benath an artificial threshold, of varying spatial frequency. To take into account the non-linear cooperative effect of noise, we have considered non-dynamical paradigm of SR used in the studies reported in [12. 13]. Nondynamical paradigm is widely applied for the study of SR in psychophysics and sensory biology [3, 12, 20].

The main results of our investigation are summarized below:

(1) We observed that at each frequency the perceived sensitivity strongly depends on noise strength and attains a peak for an intermediate strength via stochastic resonnace.

(2) The peak contrast detection sensitivity, thus attained via stochastic resonance, at each frequency varies nonmonotonically with spatial frequency.

(3) We also demonstrate that the qualitative nature of the detection sensitivity, as a function of frequency, is in good agreement with the human contrast sensitivity function measured in psychophysical experiments [21, 22]. These result indicate that the human contrast sensitivity function could be obtained in terms of a non-linear cooperative effect called SR. The observations are interesting because the elevated response was attained via SR, which was a non-linear phenomenon and also, it acted seperately on each spatial scale. Therefore, the elevated response did not gurantee the overall response as a function of frequency (contrast sensitivity function), but our results are surprisingly showing the qualitative nature of contrast sensitivity function.

Participants

\section{Materials and Methods}

Five observers with ages ranging from 25 to 50 years participated in the experiment. Two participants (authors AK and SS) were aware of the hypotheses, whereas the other four was naive to the purposes of the study. They have self-reported normal or corrected-to-normal vision. All participants were given time to familiarize themselves with the tasks prior to data collection by practicing until their responses stabilized. Best three observers' results are shown here. Written consent was obtained from each participant prior to the experiment. The experiment was approved by the Divisional Staff Committee of Applied Nuclear Physics Division, Saha Institute of Nuclear Physics.

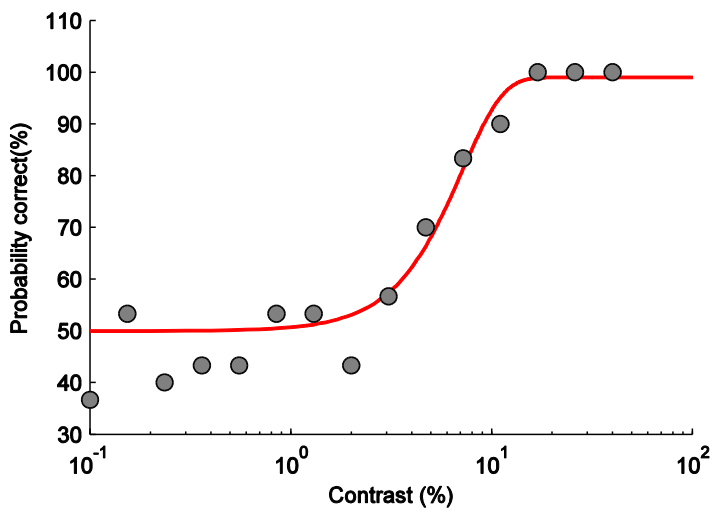


Fig. 2.Probability of correct response for SS because of 30 trials (for a particular noise level) for each of the 15 contrast levels is shown as filled circles in Fig. 2(a). The curve in red is fit of the psychometric function (Eq. (1)) to the response data. The data is taken at a noise level with power spectral density $0.0022 \mathrm{deg}^{2}$ and for $\mathrm{f}=0.5456$ cycle/deg. Contrast value corresponding to the $75 \%$ correct result is estimated from the fitted curve. In Fig 2(b), the corresponding deviance residual distribution is shown for all frequency across all observers.

Apparatus

The stimuli were presented on a NEC Multisync FP 2141 SD monitor with spatial resolution of 1024 by 768 pixels $(49.15 \mathrm{~cm}$ x $36.86 \mathrm{~cm})$ and it was refreshed at $116 \mathrm{~Hz}$. The whole procedure including Stimulus generation, presentation, and participants' responses were controlled from Dell Precision T1600 computer with ViSaGe MKII Visual Stimulus Generator from Cambridge Research System. Experiments were conducted from within Matlab, using CRS Toolbox from Cambridge Research System. The luminance measurement was performed using ColorCAL MKII Colorimeter from the same vendor. Participants performed the experiment in a dimly lit room and viewed the monitor at $750 \mathrm{~mm}$ distance binocularly.

Stimuli

Two stimuli, one contained grating plus noise and the other only noise, were presented in two separate intervals in every trial. For stimuli preparation, we have adopted a procedure similar to the one described in [12, 13]. Vertically oriented noisy sine-wave grating was input to a threshold detector (with threshold $\Delta$ ). The threshold was selected such that the grating alone (without noise contamination) becomes sub-threshold. The property of the detector was such that it produced a pulse of standard shape whenever the noisy signal crossed the threshold in the positive direction. In the absence of noise, the grating, represented by gray value $I$, being sub-threshold, could never be detected because it could never cross the barrier alone. In the presence of some additive noise $\xi$, which is usually zero mean band limited Gaussian determined by a standard deviation $\sigma$, the signal will cross the threshold at some mean frequency resulting in spikes at the detector output. The threshold crossings will determine the instantaneous rate of output spikes. The information content of the resultant output will depend on the number of spikes and its spatial distribution. In the case of grating plus noise input, the spikes and its spatial distribution at the output of the detector will embed some information of the original grating, whereas, in case of noise alone, the detector will output a train of uncorrelated spikes. In the present study, the output, in the one case, generated one of the displayed stimulus that contained grating plus noise and in the other case, the output only produced the noise stimulus. The stimuli were circular patches with diameter14.62 deg.

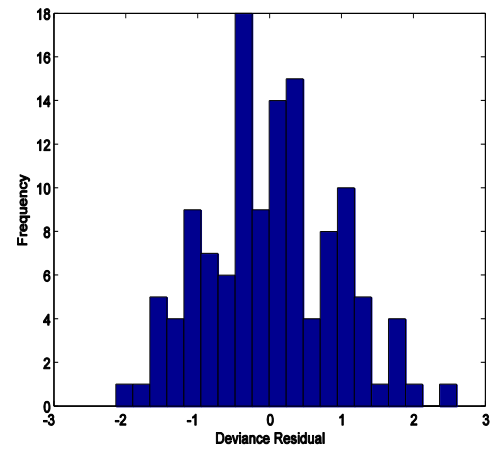

(a)

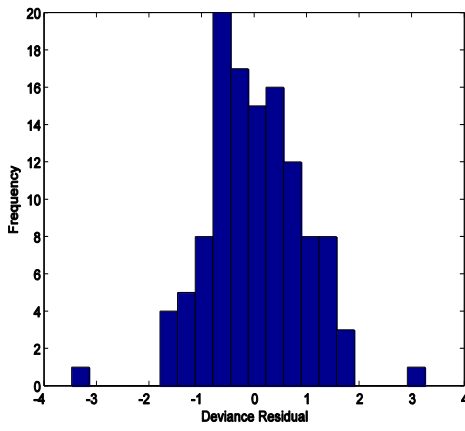

(b)

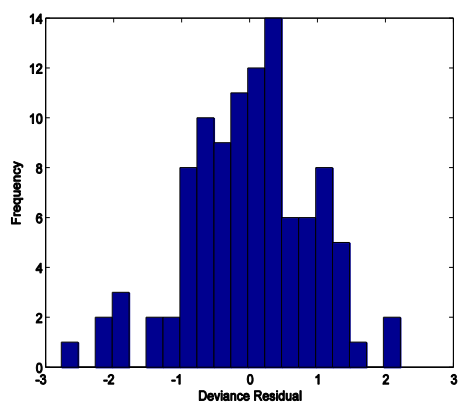

(c)

Fig. 3.Corresponding deviance residual distributions of psychometric function of different observers are shown for all frequency. (a) observer AK, (b) observer PB, (c) observer SS. 
The whole procedure was realised in the present study in the following manner. For the preparation of the stimulus that contained the grating plus noise, a sinusoidal grating image $I$ was used, which was generated by the spatial function $\mu_{0} C \sin (2 \pi f x)+\mu_{0}$, where $C$ is Michelson contrast, $f$ is the spatial frequency in cycles/deg., $\mathrm{x}$ is the horizontal coordinate in deg. and $\mu_{0}(=128)$ is the mean luminance of the display screen in 8-bit grayscale. Noise (a random number $\xi$ ) was added to the gray value $I$ in every pixel of the input image forming the noisy signal $I+\xi$. It was then threshold filtered according to the following rule: if $I+\xi>\Delta+\mu_{0}$ and the threshold is crossed in the positive direction, the gray value of that pixel was replaced with gray value 256 (white), otherwise it was replaced with 0 (black). The stimulus, therefore, consisted of only white pixels on a black background but there existed patterns resembling sine wave grating (Fig. 1).

For the generation of the stimulus, which contained only noise, the starting image was the one for which the gray values for every pixel were represented by a zero-mean Gaussian noise $\xi_{0}$ with standard deviation $\sigma_{0}$. It is then threshold filtered according to the rule: if $\xi_{0}>\Delta$ occur in the positive direction, the gray value of that pixel was replaced with 250 (white), otherwise it was replaced with 0 (black). The number of threshold crossings increased with the increase in $\sigma_{0}$. We continued increasing $\sigma_{0}$ until the number of threshold crossings equaled, with some tolerance ( $2 \%$ at the most), to that of its counterpart, the stimulus that contained grating plus noise.

Both the stimulus, therefore, consisted of only white pixels on a black background but in the signal stimulus there are patterns resembling sine grating whereas in the noise stimulus there were no pattern (Fig. 1). For particular noise strength, the visibility of the pattern depends on the original contrast level of the original sine grating. High contrast will trigger large number of threshold crossing resulting in a large number of white pixels in the signal plus noise stimulus making it easily detectable against the noisy stimulus. The number of white pixels will decrease with the decrease in contrast and at a certain contrast level, the grating plus noise stimulus will cease to be detectable with certainty. This limiting contrast was termed as threshold contrast and it was a measure of signal detection sensitivity of a participant, which we have measured for different frequencies in the present study.

\section{Procedure}

A two-interval forced-choice (2IFC) protocol with constant stimuli was used to measure the signal detection sensitivity of the participants. In this procedure, participants were presented two stationary stimuli sequentially in two separate intervals and were given the task to judge, which interval contained the grating stimulus. The trials began with a blank screen (mean luminance) in the first interval (pre-stimulus interval). The first stimulus is presented, in the second interval, on the left hand side of the screen, which was followed by another blank screen in the third interval and finally the second stimulus was presented on the right hand side of the screen in the fourth interval. The presentation schedule then waited for the users' response. The participant indicated which interval contained the signal stimulus by pressing one of the two buttons on a wireless response box. An auditory feedback was produced after each user response signifying correct (short note of $0.2 \mathrm{sec}$ duration) or incorrect response (long note of $0.5 \mathrm{sec}$ duration). A new trial began $500 \mathrm{~m}$ after the participant's response. The pre-stimulus interval, stimulus interval (SI) as well as the inter stimulus interval (ISI) were of $500 \mathrm{~ms}$ duration and a fixation point, in red, of $0.29 \mathrm{deg}$ was visible in the center of the display screen for all four intervals. For each trial, the signal stimulus was presented in one of the intervals selected at random and the other interval contained noise stimulus but the first stimulus interval was always presented at the left hand side of the display screen and the second stimulus interval was presented on the right hand side.

To obtain psychometric function, at each noise we have used 15-Michelson contrast levels for the initial sine grating that was used to generate the signal stimulus. Michelson contrast is defined as the maximal luminance minus the minimal luminance divided by their sum and thus it varied from $0(0 \%)$ to 1 (100\%).Altogether, data were recorded for 10 noise strengths and five spatial frequencies. One trial consisted of 15 recordings corresponding to 15 contrast levels for every noise strength. Contrast levels were selected in order from high to low level during stimulus presentation but the noise levels were selected randomly. Each session consisted of 450 trials; 15 trials, for 15 -contrast levels, with 30 repetitions at each noise strength. The entire session was repeated for 10 noise power spectral densities ranging between 0.0001 and $0.0028 \mathrm{deg}^{2}$, where the power spectral density is defined as the variance in luminance multiplied by the display pixel area expressed in units of $\mathrm{deg}^{2}$. Thus, a total 4500 trials were needed for each five spatial frequencies and therefore, altogether 22500 trials were recorded for each participant. 


\section{Results}

The detection sensitivity was estimated from the percent correct response, which was measured by the following expression:

$\%$ correct response $=\left[\frac{\text { Number of correct trials }}{\text { Number of trials }} \times 100\right]$. The threshold was defined as the level where the probability of correct response is midway between the $100 \%$ correct and the chance percent correct, which was $50 \%$. Therefore, the threshold level in our present study was $75 \%$. This was estimated from the fitted psychometric function given in Eq. (1) [23]

$$
\Psi(x)=(1-\delta)-(0.5-\delta) e^{-\left(\frac{x}{\mu}\right)^{\gamma}}
$$

Where $\mathrm{x}$ was the contrast of the stimulus presented and $2 \delta$ is the lapse rate. A lapse rate of $2 \%$ ( $\delta=0.01$ ) was chosen for our study. The threshold $\mu$ and the slope $\gamma$ were determined from the fitting of Eq. (1) to the response data. A typical fit of this function is shown in Fig.2. As a measure of goodness of fit, distribution of deviance residuals, described in detail in [24], across three participants is plotted in Fig. 3(a-c). It was evident from the figure; all the distribution was zero centered and Gaussian in shape. The calculation of error of all observers is more or less same.

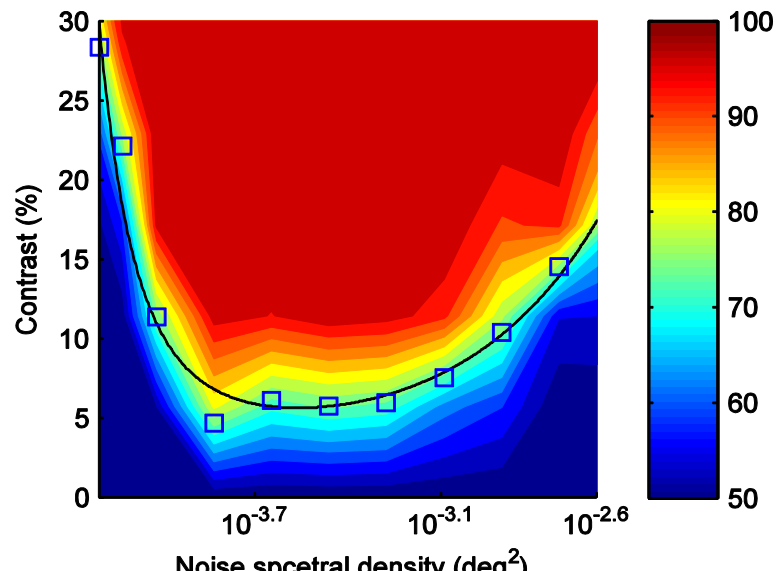

Fig. 4.Observer (SS) performance (percent correct response), obtained from psychometric function fitting, indicated by colour was plotted as function of noise spectral density (on logarithmic axis) and the contrast level. At each noise level, different colours represent percent correct response with changing contrast level. Threshold contrast (at 75\% correct response) at each noise strengths were indicated by hollow square symbols. The solid line represents fit of Eq. (2) to the threshold contrast. These data was taken at spatial frequency f=0.5456 cycle/deg.

Plot of fitted percent correct response for SS for a whole session, comprising of 4500 trials (for $\mathrm{f}=$ 0.5456 cycle/deg), was shown in Fig. 4. Each colour in the plot represents a particular value of percent correct response. It is interesting to note each colour traced a U-shaped curve in the plot. The curve also indicated that the threshold contrast (defined for 75\% correct response) first decreases with noise level, attains a minimum and then increases with further increase in noise level. This is a typical signature of SR [2] where the signal detection sensitivity of a participant (SS in the case) varies non-monotonically with noise level and attains a maximum for an intermediate noise level. Similar results were reported earlier [12-13], where participants experienced elevated contrast detection sensitivity for a non-zero amount of noise. The hollow square symbols in Fig. 4 represent the threshold contrast at 10 discrete noise levels originally used for the experiment. The minimum threshold contrast was estimated from the continuous curve (solid line) shown in Fig. 4. The solid line was obtained from the fit of Eq. (2) to the threshold contrast data (hollow squares).

$$
C_{t h}=\alpha+a \sigma_{n}^{\beta} e^{\frac{\Delta^{2}}{2 b \sigma_{n}^{2}}}
$$

Here $C_{t h}$ is the threshold contrast, $\sigma_{n}$ is standard deviation of the noise, $\Delta$ is the threshold used for stimulus generation, $\beta=2$ (chosen for the present study).The free parameters, $\alpha, a$ and $b$ were estimated from least square fitting of this equation to the contrast data. The sole purpose of this fitting was to estimate the minimum threshold contrast from the discrete set of 10 data points. A similar relation, based on the theory of level crossing detectors [20] was used in [13] for modeling the role of noise in image processing in human perceptive systems. We have modified this equation by introducing two additional free parameters $\alpha$ and $\beta$, as shown in Eq. (2). 


\section{Table 1}

Best fit parameter values in Eq. (3)

for different participants

\begin{tabular}{llll}
\hline & $K_{1}$ & $K_{2}$ & $K_{3}$ \\
\hline AK & 5.90 & 43.23 & 17.90 \\
PB & 6.27 & 36.12 & 14.19 \\
SS & 4.19 & 35.35 & 15.21 \\
\hline
\end{tabular}

These free parameters enabled better fitting to the threshold contrast data. Following a similar procedure, we have estimated the minimum threshold contrast for altogether five spatial frequencies $(\mathrm{f}=0.0369$, $0.1371,0.5456,1.0909$ and 2.0978 cycle/deg) for each participant and this was repeated for three participants. The estimated contrast detection sensitivity (inverse of minimum threshold contrast) for all participants was plotted as a function spatial frequency in Fig. 4. The plots for all participants show a typical variation with frequency, where it first rises with frequency, attains a maximum and then decreases with further increase in frequency. The solid curve, which is fit to the individual data, is the contrast sensitivity function given by [22]

$$
C S=K_{1}+K_{2}\left(0.0192+0.114 f K_{3}\right) \exp \left[-\left(0.114 f K_{3}\right)^{1.1}\right]
$$

Here $C S$ is the contrast sensitivity, $f$ is spatial frequency in cycle/deg, $K_{1}, K_{2}$ and $K_{3}$ are the fitting parameters. The original function (defined with $K_{1}=0, K_{2}=2.6$ and $K_{3}=1$ ), which represents an average nature of contrast sensitivity variation, has a peak value 1.0 at $f=8$ cycles/deg. On the contrary, in the present context the measured contrast sensitivity variation, showed in Fig. 5, peaked at a value close to $f=0.5 \mathrm{cycles} / \mathrm{deg}$ for all the participants. And additionally, to observe the relative difference between participants we did not normalize the responses and therefore, the peak values were not close to 1.0. Therefore, to make the original contrast sensitivity function reported in [22] suitable for fitting to our data, we incorporated three free parameters $K_{1}, K_{2}$ and $K_{3}$ as shown in Eq. (3). Values of the fitted parameter were reported in Table 1. It is evident from parameter values, and from the nature of sensitivity variations presented in Fig. 5, that the participants responded in a similar manner to the task of contrast detection sensitivity in psychophysical study reported here. From the goodness of fit, we can say that the elevated sensitivity agreed well with the human contrast sensitivity function.

\section{Discussions}

In the present study we have investigated the role of stochastic resonance in contrast perception, by

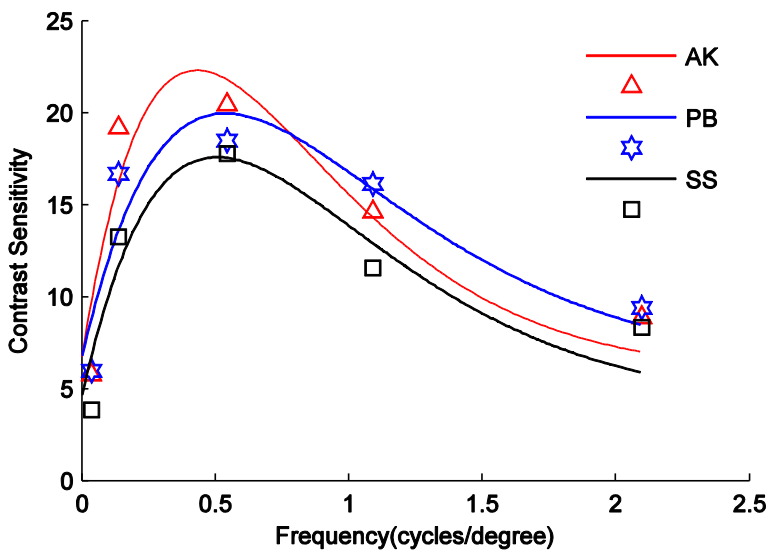

Fig. 5.Contrast sensitivity for all three observers is plotted as a function of spatial frequency. The symbols represent the measured data and solid line is the fit of the human contrast sensitivity function given in Eq. (3).

using a two-interval forced-choice (2IFC) protocol, in a visual detection task involving noisy sine grating stimuli, depressed beneath an artificial threshold, at various spatial frequencies ranging from 0.0369 $2.0978 \mathrm{cycle} / \mathrm{deg}$. of visual angle. We have measured the threshold contrast as a function of noise strength.

At each frequency, the estimated sensitivity strongly depends on the noise strength. It varies non-monotonically with noise strength and exhibits a peak at intermediate noise strength, as shown in Fig. 4, which is typical signature of SR. This kind of behavior was also observed by others [12-13]. 
We also observed a strong dependence of the perceived sensitivity on the spatial frequency. The observed peak contrast detection sensitivity, attained via stochastic resonance, varies non-monotonically with spatial frequency and reaches a maximum for an intermediate value. This behavior was consistent for all participants.

We also demonstrate that, for all participants, the qualitative nature of the detection sensitivity is in good agreement with the human contrast sensitivity function as shown in Fig. 5, which is evident from the goodness of fit of Eq. (3) (solid curves in Fig. 5) for all participants. This is an interesting result, which indicates visual systems' ability to interpret details in a sub-threshold noise contaminated stimuli varies with frequency in a manner that agree with human contrast sensitivity function.

Another important observation is that the peak of the measured contrast sensitivity curve, for all participants, is at a much lower frequency than the one usually observed. This is because the displayed stimulus, in our study, contained only white pixels (maximum contrast) on a black background. In other words, the stimulus is always displayed at maximum contrast while the frequency varied over a range of values. On the other hand, in the usual contrast sensitivity measurement, both the frequency and the contrast vary over a range. Therefore, with the stochastically elevated contrast detection sensitivity in the present context, our sensitivity to the outer world will peak at a lower frequency compared to the normal human vision.

The apparent capability of threshold crossing events to retain information of psychophysical experiments is probably due to the fact these threshold crossing events resemble spike trains that are very similar to neuron action potentials, which are produced as a result of random and weekly coherent processes [12, 20]. It has also been experimentally shown that these spike trains produced by the sensory neurons do embed lots of information $[5,6]$. These sensory neurons are noisy and in their course behavior they operate as threshold devices, which can be modeled as stochastically driven two-state process which is very similar to threshold process we have considered in our study.

\section{Conclusion}

In the present study, we have investigated the role of stochastic resonance on sensitivity in detecting in sine grating stimuli in a noisy environment following a two-interval forced-choice (2IFC) protocol. Using SR as a tool, we have estimated the threshold contrast sensitivity of our perceptual apparatus. At each frequency, the perceived sensitivity exhibits a peak at intermediate noise strength, signifying SR. We have also observed that the peak sensitivity strongly depends on spatial frequency and the dependence is in good agreement with the human contrast sensitivity function. These results indicate the utility of SR, as a tool, in human vision and suggest that this paradigm can have useful application in psychophysics experiments.

These results open up possibilities for potential application of noise in practical applications for enhancing visual perception, which can be useful as an aid for the treatment for visual impairment and for the detection of a weak signal embedded in a noisy background $[15,25]$. Further investigation in this direction is essential to fully exploit the beneficial role of noise in visual perception.

\section{References}

[1] K. Wiesenfeld and F. Moss, Stochastic resonance and the benefits of noise: from ice ages to crayfish and SQUIDs. Nature,. 373(6509). 1995, 33-36.

[2] L. Gammaitoni, P. Hänggi, P. Jung, and F. Marchesoni, Stochastic Resonance. Reviews of Modern Physics 70, $1998,223$.

[3] F. Moss, L. Ward, and W. Sannita, Stochastic resonance and sensory information processing: a tutorial and review of application. Clinical neurophysiology : official journal of the International Federation of Clinical Neurophysiology, 11 (2). 2004, 267-81.

[4] L. Ward, A. Neiman, and F. Moss, Stochastic resonance in psychophysics and in animal behavior. Biological cybernetics, 87 (2). 2002, 91-101.

[5] J. Douglass, L. Wilkens, E. Pantazelou, and F. Moss, Noise enhancement of information transfer in crayfish mechanoreceptors by stochastic resonance. Nature, 365( 6444). 1993, 337-40.

[6] J. Levin and J. Miller, Broadband neural encoding in the cricket cercal sensory system enhanced by stochastic resonance. Nature, 380(6570). 1996, 165-8.

[7] B. Gluckman, T. Netoff, E. Neel, W. Ditto, M. Spano, and S. Schiff, Stochastic Resonance in a Neuronal Network from Mammalian Brain. Physical Review Letters. 77, 1996, 4098

[8] T. Mori and S. Kai, Noise-Induced Entrainment and Stochastic Resonance in Human Brain Waves. Physical Review Letters, 88. 2002, 218101-1.

[9] M. Riani and E. Simonotto, Stochastic resonance in the perceptual interpretation of ambiguous figures: A neural network model. Physical Review Letters. 72, 1994, 3120.

[10] J. Collins, T. Imhoff, and P. Grigg, Noise-enhanced tactile sensation. Nature, 383(6603). 1996, 770.

[11] J. Collins, T. Imhoff, and P. Grigg, Noise-mediated enhancements and decrements in human tactile sensation. Physical Review E. 56, 1997, 923 .

[12] E. Simonotto, M. Riani, C. Seife, and M. Roberts..., Visual perception of stochastic resonance. Physical Review Letters, 78. 1997, 1186.

[13] M. Piana, M. Canfora, and M. Riani, Role of noise in image processing by the human perceptive system. Physical Review E. 62 (1). 2000, 195-202.

[14] K. Kitajo, D. Nozaki, L. Ward, and Y. Yamamoto, Behavioral Stochastic Resonance within the Human Brain, Physical Review Letters, 90. 2003, 218103. 
[15] H. Sasaki, M. Todorokihara, T. Ishida, J. Miyachi, T. Kitamura, and R. Aoki, Effect of noise on the contrast detection threshold in visual perception. Neuroscience letters, 408(2). 2006, 94-7.

[16] D. Russell, L. Wilkens, and F. Moss, Use of behavioural stochastic resonance by paddle fish for feeding. Nature, 402(6759). 1999, 291-4.

[17] F. Jaramillo and K. Wiesenfeld, Mechanoelectrical transduction assisted by Brownian motion: a role for noise in the auditory system. Nature neuroscience, 1(5). 1998, 384-8.

[18] E. Simonotto, F. Spano, M. Riani, A. Ferrari, and F. Levrero..., fMRI studies of visual cortical activity during noise stimulation. Neurocomputing, 26-27, 1999, 511.

[19] K. Ghosh, S. Sarkar, and K. Bhaumik, Biological Cybernetics. 100, 2009, 351.

[20] Z. Gingl, L. Kiss, and F. Moss, Non-dynamical stochastic resonance: Theory and experiments with white and arbitrarily coloured noise. Europhys. Lett.. 29 (3), 1995, 191.

[21] Campbell, F., \& Robson, J. Application of Fourier analysis to the visibility of gratings. The Journal of physiology, 197, 1968, 55166.

[22] Mannos, J., \& Sakrison, D. The effects of a visual fidelity criterion of the encoding of images. IEEE Tranctions on Information Theory. 20(4), 1974, 525-535.

[23] L. Kontsevich, C. Chen, and C. Tyler, Separating the effects of response nonlinearity and internal noise psychophysically. Vision Research, 39, 2002, 525-535.

[24] R. Goris, P. Zaenen, and J. Wagemans, Some observations on contrast detection in noise. Journal of vision, 8(9), 2008, 1-15

[25] E. Sejdić and L. Lipsitz, Necessity of noise in physiology and medicine. Computer methods and programs in biomedicine, 111(2). 2013, 459-70. 